\title{
Molecular characterization of antimicrobial resistance in Shigella sonnei isolates in Korea
}

\author{
Sung Yong Seol, Yong Tae Kim, Young Sook Jeong, Jae Young Oh, \\ Hee Young Kang, Dong Chan Moon, Jungmin Kim, Yoo Chul Lee, \\ Dong Taek Cho and Je Chul Lee
}

\begin{abstract}
Correspondence
Je Chul Lee

leejc@knu.ac.kr
\end{abstract}

Received 29 November 2005

Accepted 23 March 2006

\author{
Department of Microbiology, Kyungpook National University School of Medicine, 101, \\ Dongin-dong, Jung-gu, Daegu, 700-422, Korea
}

\begin{abstract}
The antimicrobial resistance of 122 Shigella sonnei isolates obtained in Korea during the period 1991-2000 was characterized. These isolates were highly resistant to traditional antibiotics such as trimethoprim (100\%), streptomycin (100\%), sulfamethoxazole (94\%), tetracycline (93\%) and nalidixic acid (90\%). All S. sonnei isolates carried Tn7 in their chromosomes. The $8.4 \mathrm{~kb}$ non-transferable resistance $(\mathrm{R})$ plasmid carrying tet $A$, strA-strB and sul1 was found in $93 \%$ of the $S$. sonnei isolates. Resistance to nalidixic acid first appeared in a S. sonnei isolate in 1997, and then in all S. sonnei isolates from 1998 and 1999. Resistance to commonly prescribed antibiotics such as ampicillin was increased in S. sonnei isolates during the outbreak period 1998-2000. Resistance to ampicillin was mediated by the conjugative $\mathrm{R}$ plasmids carrying b/aTEM-1. In conclusion, S. sonnei acquired antimicrobial resistance to commonly prescribed antibiotics through the horizontal transfer of conjugative R plasmids, while the genetic stability of transposon and non-transferable $\mathrm{R}$ plasmids was responsible for resistance to traditional antibiotics.
\end{abstract}

\section{INTRODUCTION}

Shigellosis remains an important cause of communityacquired gastrointestinal illness in both developing and industrialized countries. Since 1951, when Shigella sonnei was first reported to be sporadically isolated in Korea (Im \& Choi, 1961), S. sonnei has become, in the 1990s and 2000s, the primary cause of shigellosis (Kim et al., 2002; Oh et al., 2003). Shigellosis caused by S. sonnei occurred sporadically in Korea until 1997, when the annual incidence was estimated to be 2-260 cases, but large outbreaks of shigellosis caused by S. sonnei have occurred since 1998, and the annual incidence of $S$. sonnei is now estimated to be $846-2400$ cases (National Institute of Health in Korea, 2005). We previously reported that there are distinct phenotypic and genotypic differences in terms of biotypes, antimicrobial susceptibilities and PFGE profiles between the S. sonnei isolates from the 1980s and the 1990s (Jeong et al., 2003; Kim et al., 2002; Oh et al., 2003). S. sonnei isolates during the period 1977-1986 exhibited biotype a, while $S$. sonnei isolates during the period 1991-2000 exhibited biotype g. PFGE patterns of $S$. sonnei isolates during the period 1977-1986 were completely different from those of isolates during the period 1991-2000. This suggests that Korean endemic S. sonnei strains disappeared, and that new

Abbreviations: ESBL, extended-spectrum $\beta$-lactamase; R plasmid, resistance plasmid.
S. sonnei clones were introduced in the late 1980s or early 1990s.

In Korea, S. sonnei isolated during the last decade were resistant to multiple antibiotics, and a high proportion of $S$. sonnei were commonly resistant to tetracycline, streptomycin, sulfonamide and trimethoprim (Jeong et al., 2003). Additionally, antimicrobial susceptibilities of $S$. sonnei isolates gradually changed over time. The most striking changes were an increase in resistance to ampicillin and the emergence of extended-spectrum $\beta$-lactamases (ESBLs) in S. sonnei isolates. ESBLs, including TEM-15, TEM-17, TEM-19, TEM-20, TEM-52 and CTX-M-14, have been detected among recently identified $S$. sonnei isolates (Kim et al., 2004; Pai et al., 2001). However, there have been no comprehensive studies involving the molecular characterization of antimicrobial resistance in the $S$. sonnei isolates in Korea. The current study investigated the molecular mechanisms by which $S$. sonnei isolates during the period 1991-2000 were resistant to antimicrobial agents.

\section{METHODS}

Bacterial isolates. A total of 122 S. sonnei isolates were obtained from stool samples in different parts of Korea during the period 1991-2000 (Table 1). All S. sonnei isolates from sporadic shigellosis were collected. From the defined outbreaks, S. sonnei isolates that showed the same antibiogram and identical or similar PFGE pattern ( similarity value of $>95 \%$ ) were considered to be genetically 
Table 1. Transferability of antimicrobial resistance in S. sonnei isolates

Abbreviations: Tc, tetracycline; Sm, streptomycin; Su, sulfamethoxazole; Ap, ampicillin; Tp, trimethoprim; Km, kanamycin; Na, nalidixic acid; Cm, chloramphenicol; Gm, gentamicin; Cp, cefoperazone. Parentheses indicate number of strains.

\begin{tabular}{|c|c|c|c|c|c|}
\hline $\begin{array}{l}\text { Year } \\
\text { isolated }\end{array}$ & Isolation area & $\begin{array}{l}\text { No. of } \\
\text { isolates }\end{array}$ & $\begin{array}{l}\text { Antimicrobial } \\
\text { resistance }\end{array}$ & $\begin{array}{l}\text { No. of trans- } \\
\text { conjugants }\end{array}$ & $\begin{array}{l}\text { Transferable anti- } \\
\text { microbial resistance }\end{array}$ \\
\hline \multirow[t]{2}{*}{1991} & \multirow[t]{2}{*}{ Unknown (6) } & 5 & TcSmSuApTpKm & 5 & SmSuApTpKm (1), ApKm (4) \\
\hline & & 1 & SmSuApTpKm & 1 & SmSuApTpKm (1) \\
\hline 1992-1995 & $\begin{array}{l}\text { Unknown (3)/Seoul (1)/ } \\
\text { Kyungpook (1) }\end{array}$ & 5 & TcSmSuTp & 0 & - \\
\hline 1997 & Kyungpook (1) & 1 & TcSmSuTpNa & 0 & - \\
\hline \multirow[t]{3}{*}{1998} & Kyungpook (28) & 28 & TcSmSuTpNa & 0 & - \\
\hline & Kyungpook (1) & 1 & TcSmSuApTpNa & 1 & Ap (1) \\
\hline & Kyungpook (1) & 1 & $\mathrm{SmTpNa}$ & 0 & - \\
\hline \multirow[t]{7}{*}{1999} & Kyungpook (28)/Chunnam (27) & 55 & TcSmSuTpNa & 0 & - \\
\hline & Kyungpook (6) & 6 & $\mathrm{SmTpNa}$ & 0 & - \\
\hline & Kyungpook (3)/Chunnam (2) & 5 & TcSmSuApTpNa & 5 & $\begin{array}{l}\text { SmSuApTp (3), TcSmSuAp (1), } \\
\text { TcSmSu (1) }\end{array}$ \\
\hline & Chunnam (4) & 4 & TcSmSuApTpKmNa & 4 & SmSuApKm (1), ApKm (3) \\
\hline & Chunnam (3) & 3 & $\mathrm{CmTcSmSuTpNa}$ & 3 & $\mathrm{CmSmSu}(3)$ \\
\hline & Kyungpook (1) & 1 & TcSmSuApCpTpGmNa & 1 & SmSuApCpTpGm (1) \\
\hline & Kyungpook (1) & 1 & TcSmSuTpKmGmNa & 1 & SmSuTpKmGm (1) \\
\hline \multirow[t]{3}{*}{2000} & Kyungpook (2)/Chunnam (1) & 3 & TcSmSuTpNa & 0 & - \\
\hline & Chunnam (1) & 1 & TcSmSuApTpKm & 1 & ApKm (1) \\
\hline & Kyungpook (1) & 1 & TcSmSuApTpKmNa & 1 & TcSmSuApKm (1) \\
\hline
\end{tabular}

identical or closely related (Tenover et al., 1995), and a representative isolate was collected. Isolates were identified to genus and species level by conventional biochemical and serological methods (Ewing, 1986). Serological identification was performed by tube and slide agglutination with antisera (Difco Laboratories).

Antimicrobial susceptibility testing. The MICs of the antimicrobial agents were determined by the agar dilution method in accordance with the guidelines of the National Committee for Clinical Laboratory Standards (1997). The inoculated plates were incubated at $37^{\circ} \mathrm{C}$ for $20 \mathrm{~h}$. The MIC was defined as the lowest concentration of an antimicrobial agent that completely inhibited the growth of organisms. Escherichia coli ATCC 25922 and Pseudomonas aeruginosa ATCC 27853 were used as reference strains for quality control. Antimicrobial resistance was determined according to the National Committee for Clinical Laboratory Standards breakpoints, except for streptomycin, as follows: ampicillin $\left(\geqslant 32 \mathrm{mg} \mathrm{l}^{-1}\right)$, cefoperazone $\left(\geqslant 64 \mathrm{mg} \mathrm{l}^{-1}\right)$, streptomycin $\left(\geqslant 32 \mathrm{mg} \mathrm{l}^{-1}\right)$ (Voogd et al., 1992), kanamycin $\left(\geqslant 64 \mathrm{mg} \mathrm{l}^{-1}\right)$, gentamicin $\left(\geqslant 16 \mathrm{mg} \mathrm{l}^{-1}\right)$, nalidixic acid $\left(\geqslant 32 \mathrm{mg}^{-1}\right)$, ciprofloxacin $\left(\geqslant 4 \mathrm{mg} \mathrm{l}^{-1}\right)$, chloramphenicol $\left(\geqslant 32 \mathrm{mg} \mathrm{l}^{-1}\right)$, sulfamethoxazole $\left(\geqslant 512 \mathrm{mg} \mathrm{l}^{-1}\right)$, trimethoprim $\left(\geqslant 16 \mathrm{mg} \mathrm{l}^{-1}\right)$ and tetracycline $\left(\geqslant 16 \mathrm{mg} \mathrm{l}^{-1}\right)$.

Conjugal transfer of antimicrobial resistance, plasmid analysis and Southern hybridization. All $S$. sonnei isolates were included as putative donors in a conjugation experiment. E. coli RG488 Rif $^{\mathrm{r}}$ and $\mathrm{J} 53 \mathrm{Azi}^{\mathrm{r}}$ were used as recipients to detect the transfer of resistance (R) plasmids (Kim et al., 2002). Donor and recipient strains at exponential phase were grown in Trypticase soy broth (Difco Laboratories) and were mixed and incubated at $37^{\circ} \mathrm{C}$ for $20 \mathrm{~h}$. Transconjugants were selected on Mueller-Hinton agar (Difco Laboratories) supplemented with the combination of rifampicin $\left(50 \mu \mathrm{g} \mathrm{ml}^{-1}\right)$ or sodium azide $\left(50 \mu \mathrm{g} \mathrm{ml}^{-1}\right)$, and with tetracycline $\left(20 \mu \mathrm{g} \mathrm{ml}^{-1}\right)$, streptomycin $\left(32 \mu \mathrm{g} \mathrm{ml}^{-1}\right)$, trimethoprim $(50 \mu \mathrm{g}$ $\left.\mathrm{ml}^{-1}\right)$ or ampicillin $\left(50 \mu \mathrm{g} \mathrm{ml}^{-1}\right)$. Plasmid DNAs from clinical isolates or their transconjugants were isolated by the alkaline extraction method (Birnboim \& Doly, 1979). Plasmid DNAs were digested with EcoRI or SmaI restriction enzymes (Boehringer Mannheim) and separated by electrophoresis in $0.8 \%$ agarose gels. For Southern hybridization, the denatured DNAs were transferred to a positively charged nylon membrane and hybridized with the probes. The probes were labelled with digoxigenin-11-dUTP by random labelling methods (Boehringer Mannheim). A digoxigenin DNA labelling and detection kit was used according to the manufacturer's instructions.

PFGE. Genomic DNA was digested with XbaI (Boehringer Mannheim) for $20 \mathrm{~h}$ and separated in a $1 \%$ agarose gel with a contour-clamped homogeneous electric field apparatus (CHEF-DR III, Bio-Rad). The conditions for electrophoresis were $6 \mathrm{~V} \mathrm{~cm}^{-1}$ for $21 \mathrm{~h}$, with the pulse time increasing from 5 to $40 \mathrm{~s}$ (Kim et al., 2002).

PCR amplification of integrons and Tn3. To determine the presence of class 1 and class 2 integrons, PCR amplification of intI1 and intI2 was performed, respectively, as described previously (Oh et al., 2002). The gene cassette regions of the class 1 and class 2 integrons were amplified using primer pairs hep58/hep59 and hep74/ hep51, respectively (White et al., 2000, 2001). To determine identical arrays of gene cassettes, same-sized amplicons were digested with RsaI and HinfI (Boehringer Mannheim). Every PCR product of cassette regions that showed different restriction patterns was ligated with a pGEM-T Easy vector (Promega) and transformed to E. coli $\mathrm{DH} 5 \alpha$ cells. Sequencing reactions were performed with a doublestranded plasmid preparation using dideoxy chain termination with T7 and Sp6 primers. Tn3-specific regions, an internal fragment of $\operatorname{tnpR}$, were amplified using the Expand Long Template PCR system (Boehringer Mannheim) with a specific primer (5'-GTCTGACGCTCAGTGGAACG-3') (Jeong et al., 2003).

PCR amplification of antimicrobial resistance genes. PCR was performed to amplify a specific antimicrobial resistance gene for 


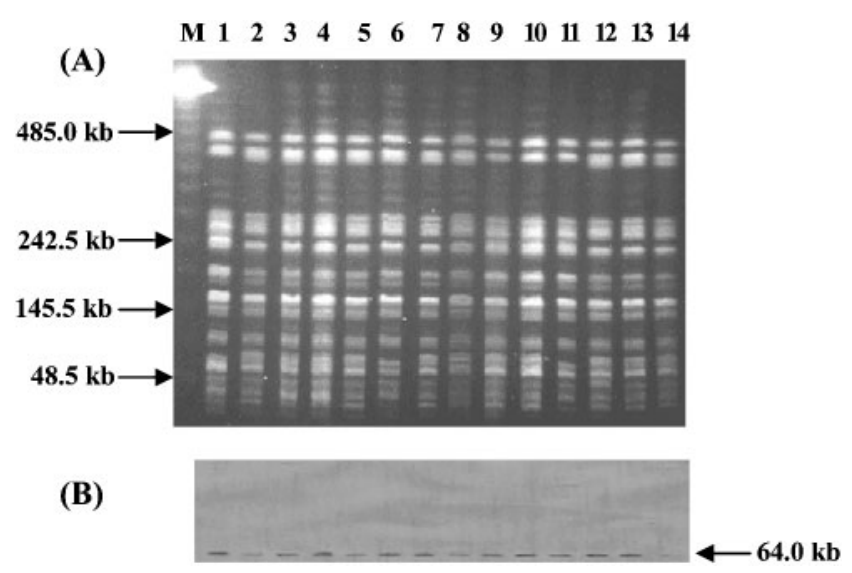

Fig. 1. PFGE patterns of $X b a l$-digested genomic DNA of $S$. sonnei isolates $(A)$ and Southern blot of gel hybridized with a dfrA1 gene probe (B). Lanes: $M$, lambda ladder (Bio-Rad); 1, H002 from 1991; 2, H003 from 1991; 3, H012 from 1995; 4, H021 from 1997; 5, H022 from 1998; 6, H025 from 1998; 7, H043 from 1998; 8, H101 from 1999; 9, H115 from 1999; 10, H265 from 2000; 11, H129 from 1999; 12, H206 from 1999; 13, H152 from 2000, 14, H259 from 2000.

each antimicrobial resistance. Primers and amplification conditions for $b l a_{\text {TEM }}$ (Lee et al., 2001b) and $d f r$ (Lee et al., 2001a) were as described previously. Amplification of the specific resistance genes was performed with the following primer sets: strA-F (5'-ATG GTG GAC CCT AAA ACT CT-3') and strB-R (5'-CGT CTA GGA TCG AGA CAA AG-3') for $s t r A-s t r B$, APH3' ${ }^{\prime}-\mathrm{F}\left(5^{\prime}\right.$-GCA TTT TAT CCG TAC TCC TG-3') and APH3'-R (5'-AAC CTA TTA ATT TCC CCT CG-3') for $a p h 3^{\prime}$, tetA-F (5'-GGC CAG ACG TGA AAC CCA ACA$\left.3^{\prime}\right)$ and tetA-R (5'-CGC GAA GGC AAG CAG GAT G-3') for tetA, sul1-F (5'-CTT CGA TGA GAG CGG CGG C-3') and sull-R (5'-CGC AAG GCG GAA ACC CGC GCC-3') for sull, and sul2-F (5'-TCA TTT TCG GCA TCG TCA ACA-3') and sul2-R (5'-GCG CGA AAC AGA CAG AAG CAC-3') for sul2. All PCR amplifications were performed using the following amplification scheme: one cycle of denaturation at $94{ }^{\circ} \mathrm{C}$ for $5 \mathrm{~min}$, followed by 30 cycles of denaturation at $94^{\circ} \mathrm{C}$ for $1 \mathrm{~min}$, annealing at $58^{\circ} \mathrm{C}$ for $1 \mathrm{~min}$, and extension at $72{ }^{\circ} \mathrm{C}$ for $1 \mathrm{~min}$, with a final extension at $72{ }^{\circ} \mathrm{C}$ for $10 \mathrm{~min}$.

\section{RESULTS}

\section{Transferable antimicrobial resistance of S. sonnei isolates}

S. sonnei isolated during the period 1991-2000 showed very similar PFGE patterns (Fig. 1A), but 10 different antimicrobial resistance patterns were detected (Table 1). To determine whether antimicrobial resistance could be transferred, conjugal transfer of R plasmids was performed. Of the $122 \mathrm{~S}$. sonnei isolates tested, $22(18.0 \%)$ isolates transferred their antimicrobial resistance to an E. coli strain (Table 1). Twenty-one S. sonnei isolates transferred one $\mathrm{R}$ plasmid, while one isolate from 1999 (H223) transferred two different $\mathrm{R}$ plasmids. Eleven different antimicrobial resistance patterns were obtained among the 23 transconjugants. Resistance to ampicillin, gentamicin and kanamycin was transferred to an E. coli recipient, except for one ampicillin-resistant $S$. sonnei isolate from 1999. Resistance to tetracycline, trimethoprim, streptomycin and sulfamethoxazole was transferred to an E. coli strain in $2.5 \%$ (3/122), $4 \cdot 1 \%(5 / 122), 8 \cdot 2 \%(10 / 122)$ and $8 \cdot 2 \%(10 / 122)$ of $S$. sonnei isolates, respectively.

\section{Molecular characterization of conjugative $\mathbf{R}$ plasmids}

Of the 23 transconjugants that transferred antimicrobial resistance, the conjugative $\mathrm{R}$ plasmids were classified into 11 groups by their RFLP patterns, antimicrobial resistance patterns, presence of Tn 3 , class 1 integrons and antimicrobial resistance genes (Table 2). The transconjugants that transferred antimicrobial resistance carried one large plasmid of $70-80 \mathrm{~kb}$; the RFLP patterns of conjugative R plasmids were different (Fig. 2). To determine whether the

Table 2. Characterization of conjugative $\mathrm{R}$ plasmids in $\mathrm{S}$. sonnei isolates

For abbreviations, see Table 1.

\begin{tabular}{|c|c|c|c|c|c|}
\hline Plasmid (isolate) & $\begin{array}{l}\text { No. of } \\
\text { isolates }\end{array}$ & $\begin{array}{l}\text { Antimicrobial } \\
\text { resistance }\end{array}$ & $\begin{array}{c}\text { Presence } \\
\text { of } \operatorname{Tn} 3\end{array}$ & $\begin{array}{l}\text { Gene cassettes of } \\
\text { class } 1 \text { integron }\end{array}$ & Resistance genes \\
\hline pKY032 (H003) & 2 & SmSuApTpKm & + & aadA2-orfF-dfrA12 & aadA2, sul1, bla $a_{\mathrm{TEM}-1}, d f r A 12, a p h 3^{\prime}$ \\
\hline pKY1101 (H110) & 1 & SmSuApKm & - & - & strA-strB, sul2, bla $a_{\mathrm{TEM}-1}, a p h 3^{\prime}$ \\
\hline pKY1381 (H138) & 3 & SmSuApTp & + & aadA2-orfF-dfrA12 & strA-strB, aadA2, sul1, bla $a_{\mathrm{TEM}-1}, d f r A 12$ \\
\hline pKY2061 (H206) & 1 & SmSuTpKmGm & - & aadA2-orfF-dfrA12 & strA-strB, aadA2, sul1, dfrA12, aph3' \\
\hline pKY2131 (H213) & 1 & TcSmSuAp & - & - & $\operatorname{tet} A, \operatorname{str} A-s t r B$, sul $2, b l a_{\mathrm{TEM}-1}$ \\
\hline pKY2191 (H219) & 3 & $\mathrm{CmSmSu}$ & - & - & strA-strB, sul2 \\
\hline pKY2231 (H223) & 1 & $\mathrm{TcSmSu}$ & - & $\operatorname{aadA1}$ & $\operatorname{tet} A, \operatorname{str} A-s \operatorname{tr} B, \operatorname{aad} A$, sull \\
\hline pKY2654 (H265) & 8 & ApKm & + & - & $b l a_{\mathrm{TEM}-1}, a p h 3^{\prime}$ \\
\hline
\end{tabular}




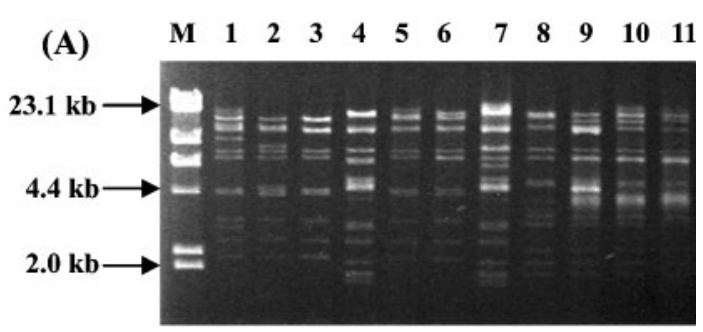

(B)

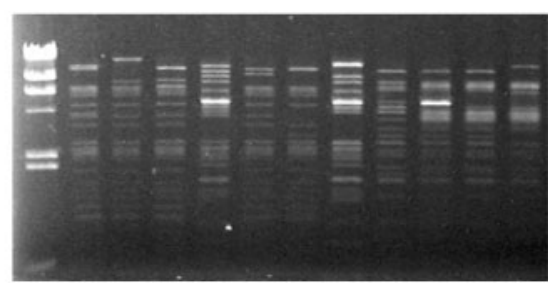

Fig. 2. Restriction endonuclease analysis of $R$ plasmids extracted from transconjugants by EcoRI (A) and Smal (B). Lanes: M, lambda DNA/Hindlll marker; 1, pKY032 from H003; 2, pKY1402 from H140; 3, pKY011 from H001; 4, pKY1101 from $\mathrm{H} 110$; 5, pKY1381 from $\mathrm{H} 138$; 6, pKY2322 from $\mathrm{H} 232$; 7, pKY2131 from H213; 8, pKY062 from H006; 9, pKY1292 from $\mathrm{H} 129$; 10, pKY1302 from $\mathrm{H} 130 ; 11$, pKY2654 from $\mathrm{H} 265$.

conjugative R plasmids carried class 1 or class 2 integrons, the intl1 and intl2 genes were amplified. Of the 11 conjugative R plasmids, five carried class 1 integrons. No class 2 integrons were found in any of the transconjugants. Among the five conjugative $\mathrm{R}$ plasmids carrying class 1 integrons, four carried a gene cassette of $d f r A 12$-orfF-aadA2 and one carried aadA1. A class 1 integron carrying dfrA12-orfFaadA2 was found in S. sonnei isolates from 1991, 1999 and 2000, while a class 1 integron carrying aadA1 was found in the isolate from 1999.

The mechanisms by which the transconjugants were resistant to antimicrobial agents were determined. Resistance to tetracycline, kanamycin, ampicillin and trimethoprim was mediated by tet $A, a p h 3^{\prime}, b l a_{\mathrm{TEM}-1}$ and $d f r A 12$, respectively (Table 2). Resistance to cefoperazone was mediated by $b l a_{\text {TEM-52 }}$ in one S. sonnei isolate from 1999 (Jeong et al., 2003). Resistance to sulfamethoxazole was mediated by sull in the five class 1 integron-carrying R plasmids, and sul2 in the four R plasmids that did not contain class 1 integrons. Resistance to streptomycin was mediated by $\operatorname{str} A-s \operatorname{tr} B$, aadA2 or aadA1.

\section{Molecular characterization of non-transferable antimicrobial resistance}

To characterize the non-transferable antimicrobial resistance among $S$. sonnei isolates, PCR for $d f r A 1, d f r A 5, d f r A 7$, $d f r A 8, d f r A 12, d f r A 13$ and $d f r A 17$ was first performed (Lee et al., 2001b). Southern hybridization of the PFGE gel with a $d f r A 1$ probe showed that all $122 \mathrm{~S}$. sonnei isolates carried dfrA1 in their chromosomes (Table 3, Fig. 1B). To characterize the association of $d f r A 1$ genes with $\mathrm{Tn} 7$, intI2 and

Table 3. Characterization of non-transferable antimicrobial resistance in S. sonnei isolates

For abbreviations, see Table 1.

\begin{tabular}{|c|c|c|c|c|}
\hline \multirow{2}{*}{$\begin{array}{l}\text { Year } \\
\text { isolated }\end{array}$} & \multirow{2}{*}{$\begin{array}{l}\text { No. of } \\
\text { isolates }\end{array}$} & \multirow{2}{*}{$\begin{array}{l}\text { Antimicrobial } \\
\text { resistance }\end{array}$} & \multicolumn{2}{|c|}{ Antimicrobial resistance genes located in: } \\
\hline & & & Chromosome & $\begin{array}{l}\text { Non-transferable } \\
\text { R plasmid }\end{array}$ \\
\hline \multirow[t]{2}{*}{1991} & 5 & TcSmSuApTpKm & $\operatorname{Tn} 7($ dfrA1-sat-aadA1) & tet $A$, strA-strB, sul1 \\
\hline & 1 & SmSuApTpKm & $\operatorname{Tn} 7(d f r A 1-s a t-a a d A 1)$ & - \\
\hline $1992-1995$ & 5 & TcSmSuTp & $\operatorname{Tn} 7$ (dfrA1-sat-aadA1) & tet $A, \operatorname{str} A-s \operatorname{tr} B$, sull \\
\hline 1997 & 1 & TcSmSuTpNa & $\operatorname{Tn} 7$ (dfrA1-sat-aadA1) & tet $A$, strA-strB, sull \\
\hline \multirow[t]{3}{*}{1998} & 28 & TcSmSuTpNa & $\operatorname{Tn} 7(d f r A 1-$ sat-aadA1) & tet $A, \operatorname{str} A-\operatorname{str} B$, sull \\
\hline & 1 & TcSmSuApTpNa & $\operatorname{Tn} 7($ dfrA1-sat-aadA1) & tet $A$, strA-strB, sul1 \\
\hline & 1 & $\mathrm{SmTpNa}$ & $\operatorname{Tn} 7($ dfrA1-sat-aadA1) & - \\
\hline \multirow[t]{7}{*}{1999} & 55 & TcSmSuTpNa & $\operatorname{Tn} 7$ (dfrA1-sat-aadA1) & tet $A, \operatorname{str} A-s \operatorname{tr} B$, sull \\
\hline & 6 & $\mathrm{SmTpNa}$ & $\operatorname{Tn} 7(d f r A 1-s a t-a a d A 1)$ & - \\
\hline & 5 & TcSmSuApTpNa & $\operatorname{Tn} 7(d f r A 1-$ sat-aadA1) & tet $A, \operatorname{str} A-s \operatorname{tr} B$, sull \\
\hline & 4 & TcSmSuApTpKmNa & $\operatorname{Tn} 7(d f r A 1-$ sat-aadA1) & tet $A, \operatorname{str} A-s \operatorname{tr} B$, sull \\
\hline & 3 & CmTcSmSuTpNa & $\operatorname{Tn} 7(d f r A 1-$ sat-aadA1) & tet $A, \operatorname{str} A-\operatorname{str} B$, sull \\
\hline & 1 & TcSmSuApCpTpGmNa & $\operatorname{Tn} 7(d f r A 1-$ sat-aadA1) & tet $A, \operatorname{str} A-s \operatorname{tr} B$, sul1 \\
\hline & 1 & TcSmSuTpKmGmNa & $\operatorname{Tn} 7(d f r A 1-$ sat-aadA1) & tet $A, \operatorname{str} A-\operatorname{str} B$, sul1 \\
\hline \multirow[t]{3}{*}{2000} & 3 & TcSmSuTpNa & $\operatorname{Tn} 7(d f r A 1-$ sat-aadA1) & tet $A, \operatorname{str} A-s \operatorname{tr} B$, sull \\
\hline & 1 & TcSmSuApTpKm & $\operatorname{Tn} 7(d f r A 1-$ sat-aadA1) & tet $A, \operatorname{str} A-s \operatorname{tr} B$, sull \\
\hline & 1 & TcSmSuApTpKmNa & $\operatorname{Tn} 7(d f r A 1-s a t-a a d A 1)$ & - \\
\hline
\end{tabular}


gene cassette regions of class 2 integrons, a $440 \mathrm{bp}$ fragment of intI 2 and $2224 \mathrm{bp}$ of a class 2 integron, were amplified and sequenced. All $S$. sonnei isolates carried $d f r A l$ as a gene cassette of $\operatorname{Tn} 7, d f r A 1$-sat-aadA1.

Of the 122 S. sonnei isolates tested, $113(92 \cdot 6 \%)$ isolates carried $8.4 \mathrm{~kb}$ of non-transferable R-plasmid DNA, as demonstrated by a plasmid profile and conjugation experiment (Fig. 3A, Table 3). To characterize the $8.4 \mathrm{~kb}$ nontransferable R plasmid, plasmid DNA was extracted from S. sonnei H111, which was resistant to tetracycline, streptomycin, sulfamethoxazole, trimethoprim and nalidixic acid, and introduced into E. coli $\mathrm{DH} 5 \alpha$ by transformation. The transformant carrying the $8.4 \mathrm{~kb}$ non-transferable $\mathrm{R}$ plasmid exhibited resistance to tetracycline, streptomycin and sulfamethoxazole, which were mediated by tetA, strA-strB and sull, respectively. The antimicrobial resistance genes and restriction map of the $8.4 \mathrm{~kb}$ of non-transferable $\mathrm{R}$ plasmid in the current study were identical to the GenBank entry for pKKTET7 of the S. sonnei isolate from 1998 in Korea (AF497970). To determine whether 113 S. sonnei isolates carried tet $A, \operatorname{str} A$-strB and sull in the $8 \cdot 4 \mathrm{~kb}$ of nontransferable R plasmid, PCR amplification and Southern hybridization were performed, which revealed that tet $A$, str $A$-strB and sull were present and encoded in the $8.4 \mathrm{~kb}$ of non-transferable $\mathrm{R}$ plasmid (Fig. 3B, Table 3). It was concluded that the $8.4 \mathrm{~kb}$ of non-transferable R plasmid was responsible for the high prevalence of resistance to these agents in S. sonnei isolates in Korea.

Resistance to nalidixic acid first appeared in a $S$. sonnei isolate in 1997, and then in all S. sonnei isolates from 1998 and 1999 (Table 1). However, nalidixic acid-susceptible $S$. sonnei isolates reappeared in a defined outbreak of shigellosis in 2000 .

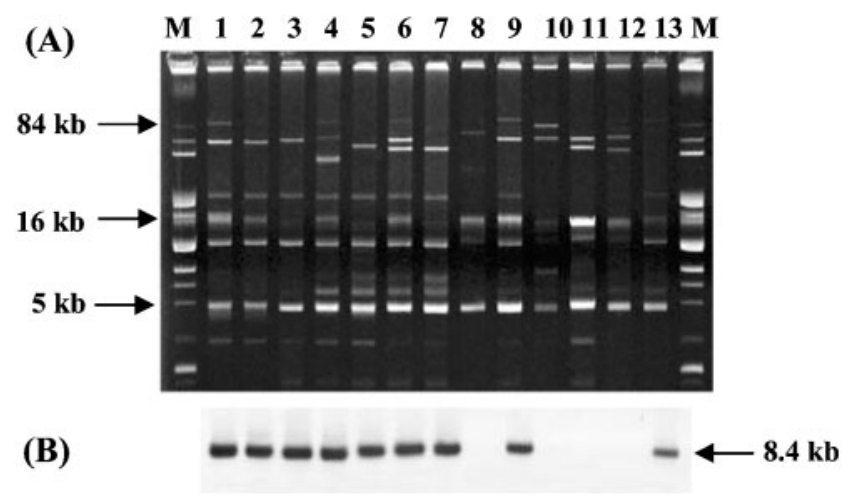

Fig. 3. Plasmid profiles of $S$. sonnei isolates $(A)$ and Southern blot of gel hybridized with tet $A$ gene (B). Lanes: $M$, size marker from E. coli KE327; 1, H008 from 1992; 2, H002 from 1991; 3, H012 from 1995; 4, H022 from 1998; 5, H025 from 1998; 6, H101 from 1999; 7, H111 from 1999; 8, H003 from 1991; 9, H152 from 2000; 10, H043 from 1998; 11, H115 from 1999; 12, H151 from 2000; 13, H210 from 1999.

\section{DISCUSSION}

The current study demonstrated that $S$. sonnei isolates have acquired resistance to ampicillin through conjugative $\mathrm{R}$ plasmids carrying $b l_{\mathrm{TEM}-1}$, while the $8.4 \mathrm{~kb}$ of nontransferable R plasmid carrying tetA, strA-strB and sull is responsible for resistance to tetracycline, streptomycin and sulfamethoxazole. Resistance genes for ampicillin, gentamicin and kanamycin, except for one ampicillin-resistance gene, were all located in the conjugative R plasmids. However, resistance genes for trimethoprim and sulfamethoxazole were mainly located in $\operatorname{Tn} 7$ in the chromosome and in the $8.4 \mathrm{~kb}$ non-transferable R plasmid, respectively. Some of the resistance genes for trimethoprim and sulfamethoxazole were also found in the conjugative $\mathrm{R}$ plasmids as a gene cassette of class 1 integrons.

Bacteria can acquire antimicrobial resistance when they are confronted with antibiotic selective pressures. The most commonly encountered resistance mechanism among Enterobacteriaceae is the acquisition of antimicrobial resistance genes through mobile genetic elements, including $\mathrm{R}$ plasmids and transposons. Ampicillin has been the most commonly used antibiotic for treatment of shigellosis in Korea during the last two decades. Resistance to ampicillin was found in all six S. sonnei isolates from 1991, but not in the isolates from 1992 to 1997; thereafter, resistance to ampicillin reappeared during the outbreak period 19982000 (Table 1). All ampicillin-resistant S. sonnei isolates carried the conjugative R plasmids harbouring $b l a_{\mathrm{TEM}-1}$. This finding indicates that $S$. sonnei can acquire resistance to commonly prescribed antibiotics through conjugative $\mathrm{R}$ plasmids.

Since the first emergence of resistance to trimethoprim in S. sonnei isolates from 1978 in Korea (Chun \& Seol, 1979), resistance to trimethoprim has been detected in $94 \%$ of S. sonnei isolates from the 1980 s and in $100 \%$ of isolates from the 1990s and 2000s. Accordingly, trimethoprim combined with sulfamethoxazole (co-trimoxazole) is no longer recommended in Korea for treatment of shigellosis. In the current study, resistance to trimethoprim was mediated by $d f r A 1$ and/or $d f r A 12$. $d f r A 1$ was located in the chromosome as a gene cassette of $\operatorname{Tn} 7$, while $d f r A 12$ was found as a gene cassette of class 1 integrons in the conjugative R plasmids. $\mathrm{Tn} 7$ was not detected in the $S$. sonnei isolates from the $1980 \mathrm{~s}$, while $\operatorname{Tn} 7$ was detected in all $S$. sonnei isolates from the 1990s and 2000s (Kim et al., 2002). This suggests that the S. sonnei strains carrying $\mathrm{Tn} 7$ in the chromosome were introduced at this time and widely disseminated in all parts of Korea. To date, five class 1 integrons carrying $d f r$ genes, dfrA1-unknown ORF, dfrA1-aadA2, dfrA12-orfF-aadA2, $d f r A 17-a a d A 5$ and aacA4-catB4-dfrA1-unknown gene, have been detected among Enterobacteriaceae in Korea (Yu et al., 2003). However, gene cassettes of $d f r A 12$-orfF-aadA2 were identified in the $S$. sonnei isolates tested. It would seem that $S$. sonnei has selected class 1 integrons carrying $d f r A 12$ orfF-aadA2, thereby accounting for the occurrence of dfrA12. 
In addition to trimethoprim, sulfamethoxazole is one of the traditional antibiotics used to treat bacterial infection. Since resistance to sulfamethoxazole was reported to be found in both S. sonnei and Shigella flexneri isolates from the early 1970s in Korea (Oh \& Chun, 1977), single therapy with sulfamethoxazole has not been recommended to treat shigellosis. In the current study, $94 \%$ of S. sonnei isolates were resistant to sulfamethoxazole, which was mediated by sul1 and/or sul2. Of the 115 sulfamethoxazole-resistant $S$. sonnei isolates, sul1 was detected in the $114 \mathrm{~S}$. sonnei isolates, while sul 2 was detected in six isolates carrying conjugative $\mathrm{R}$ plasmids. Accordingly, resistance to sulfamethoxazole was mainly mediated by sull, which was located in the $8.4 \mathrm{~kb}$ of non-conjugative $\mathrm{R}$ plasmid.

We inferred that Korean endemic S. sonnei strains disappeared in the late 1980s and that a new S. sonnei clone was first introduced to Korea between the late 1980s and early 1990s (Jeong et al., 2003; Kim et al., 2002; Oh et al., 2003). The new clone of $S$. sonnei exhibited biotype $\mathrm{g}$ and a characteristic PFGE pattern (Fig. 1A). They carried $8.4 \mathrm{~kb}$ of non-transferable R plasmid and were commonly resistant to tetracycline, streptomycin, sulfamethoxazole, trimethoprim and nalidixic acid. Among the six $S$. sonnei isolates from 1991, five carried $8.4 \mathrm{~kb}$ of non-transferable plasmid DNA. This was the first appearance of the $8.4 \mathrm{~kb}$ of non-transferable plasmid in the new clone of $S$. sonnei. Thereafter, all $S$. sonnei isolates tested, except for eight isolates, carried this non-transferable $\mathrm{R}$ plasmid. This suggests that the $8.4 \mathrm{~kb}$ of non-transferable plasmid is genetically stable in $S$. sonnei strains.

An increase in the use of first-line antibiotics, such as ampicillin and co-trimoxazole, for shigellosis might be responsible for the acquisition of resistance to these antibiotics in $S$. sonnei isolates. The acquisition of conjugative $\mathrm{R}$ plasmids carrying $b l_{\text {TEM-1 }}$ was responsible for resistance to ampicillin. Tn7 in the chromosome was responsible for resistance to trimethoprim and streptomycin. The acquisition of the $8.4 \mathrm{~kb}$ of non-transferable $\mathrm{R}$ plasmid was responsible for resistance to tetracycline, streptomycin and sulfamethoxazole. Accordingly, combinations of three genetic repertoires, conjugative $\mathrm{R}$ plasmids, $\mathrm{Tn} 7$ and the $8.4 \mathrm{~kb}$ of non-transferable $\mathrm{R}$ plasmid, can cover resistance to the antibiotics generally used for treatment of shigellosis. Because S. sonnei has undergone evolutionary changes in antimicrobial resistance over time, an appropriate antimicrobial therapy will be necessary for treatment of shigellosis to prevent the emergence of a new type of antimicrobial resistance or multiresistance. The diversity of ESBL genes in S. sonnei strains is an example of the evolutionary changes in antimicrobial resistance.

\section{ACKNOWLEDGEMENTS}

This study was supported by a Grant of the Korea Health 21 R\&D Project, Ministry of Health and Welfare, Republic of Korea
(03-PJ1-PG1-CH03-0002). We thank Tae Sook Kim and Hwa Sook Shon, from the Department of Microbiology, Kyungpook National University School of Medicine, for technical assistance.

\section{REFERENCES}

Birnboim, I. C. \& Doly, J. (1979). A rapid alkaline extraction procedure for screening recombinant plasmid DNA. Nucleic Acids Res 7, 1513-1523.

Chun, D. \& Seol, S. Y. (1979). Drug resistance of Shigella and Salmonella and the inhibition and elimination of drug resistance. J Korean Soc Microbiol 14, 27-37.

Ewing, W. H. (1986). Edwards and Ewing's Identification of Enterobacteriaceae, 4th edn. New York: Elsevier.

Im, Y. H. \& Choi, K. D. (1961). Studies on the Shigella isolated in Southern Korea with reference to non-Shigella strains possessing Shigella antigens. Showa Med J 20, 1-8.

Jeong, Y. S., Lee, J. C., Kang, H. Y. \& 7 other authors (2003). Epidemiology of nalidixic acid resistance and TEM-1- and TEM-52mediated ampicillin resistance of Shigella sonnei isolates obtained in Korea between 1980 and 2000. Antimicrob Agents Chemother 47, 3719-3723.

Kim, K. S., Oh, J. Y., Jeong, Y. W., Cho, J. W., Park, J. C., Cho, D. T. \& Lee, J. C. (2002). Epidemiological typing and characterization of $d f r$ genes of Shigella sonnei isolates in Korea during the last two decades. J Microbiol Biotechnol 12, 106-113.

Kim, S., Kim, J., Kang, Y., Park, Y. \& Lee, B. (2004). Occurrence of extended-spectrum $\beta$-lactamases in members of the genus Shigella in the Republic of Korea. J Clin Microbiol 42, 5264-5269.

Lee, J. C., Oh, J. Y., Cho, J. W., Park, J. C., Kim, J. M., Seol, S. Y. \& Cho, D. T. (2001a). The prevalence of trimethoprim-resistanceconferring dihydrofolate reductase genes in urinary isolates of Escherichia coli in Korea. J Antimicrob Chemother 47, 599-604.

Lee, J. C., Oh, J. Y., Kim, K. S., Jeong, Y. W., Cho, J. W., Park, J. C., Seol, S. Y. \& Cho, D. T. (2001b). Antimicrobial resistance of Shigella sonnei in Korea during the last two decades. APMIS 109, 228-234.

National Committee for Clinical Laboratory Standards (1997). Methods for Dilution Antimicrobial Susceptibility Tests for Bacteria that Grow Aerobically. Approved standard M7-A4. Wayne, PA: National Committee for Clinical Laboratory Standards.

National Institute of Health in Korea (2005). The Annual Frequency of Shigellosis in Korea. http://dis.cdc.go.kr/statistics/Statistics_ Disease_Year.asp (in Korean).

Oh, S. Y. \& Chun, D. (1977). Antimicrobial resistance and R plasmids in Shigella. Korean J Internal Med 20, 591-596.

Oh, J. Y., Kim, K. S., Jeong, Y. W., Cho, J. W., Park, J. C. \& Lee, J. C. (2002). Epidemiological typing and prevalence of integrons in multiresistant Acinetobacter strains. APMIS 110, 247-252.

Oh, J. Y., Yu, H. S., Kim, S. K., Seol, S. Y., Cho, D. T. \& Lee, J. C. (2003). Changes in patterns of antimicrobial susceptibility and integron carriage among Shigella sonnei isolates from southwestern Korea during epidemic periods. J Clin Microbiol 41, 421-423.

Pai, H., Choi, E., Lee, H., Hong, J. Y. \& Jacoby, G. A. (2001). Identification of CTX-M-14 extended-spectrum $\beta$-lactamase in clinical isolates of Shigella sonnei, Escherichia coli, and Klebsiella pneumoniae in Korea. J Clin Microbiol 39, 3747-3749.

Tenover, F. C., Arbeit, R. D., Goering, R. V., Mickelsen, P. A., Murray, B. E., Persing, D. H. \& Swaminathan, B. (1995). Interpreting chromosomal DNA restriction patterns produced by pulsed-field gel electrophoresis: criteria for bacterial strain typing. J Clin Microbiol 33, 2233-2239. 
Voogd, C. E., Schot, C. S., van Leeuwen, W. J. \& van Klingeren, B. (1992). Monitoring of antibiotic resistance in Shigella isolated in the Netherlands 1984-1989. Eur J Clin Microbiol Infect Dis 11, 164-167.

White, P. A., Mclver, C. J., Deng, Y. M. \& Rawlinson, W. D. (2000). Characterization of the two gene cassettes, aadA5 and dfrA17. FEMS Microbiol Lett 182, 265-269.
White, P. A., Mclver, C. J. \& Rawlinson, W. D. (2001). Integrons and gene cassettes in the Enterobacteriaceae. Antimicrob Agents Chemother 45, 2658-2661.

Yu, H. S., Lee, J. C., Kang, H. Y. \& 9 other authors (2003). Changes in gene cassettes of class 1 integrons among Escherichia coli isolates from urine specimens collected in Korea during the last two decades. J Clin Microbiol 41, 5429-5433. 